\title{
EFEITO INERCIAL MICROSCÓPICO NO ESTUDO DA TRANSIÇÃO DO ESCOAMENTO IMCOMPRESSÍVEL NÃO- DARCIANO EM MEIO POROSO NÃO CONSOLIDADO
}

\author{
H.E.MACIEL ${ }^{1}$ e L.D.T.CÂMARA ${ }^{1 *}$ \\ ${ }^{1}$ Instituto Politécnico da Universidade do Estado do Rio de Janeiro (IPRJ-UERJ), \\ Departamento de Engenharia Mecânica e Energia-DEMEC \\ *E-mail para contato: dcamara@iprj.uerj.br
}

\begin{abstract}
RESUMO - Muitas das situações de escoamento de fluidos em meios porosos estão relacionadas a velocidades que remetem a condições não-darcianas. Diversos grupos vêm trabalhando de forma a compreender os aspectos da transição de escoamento de fluidos em meios porosos. Um aparato experimental foi implementado, neste trabalho, de forma a estudar o escoamento de água em um meio poroso não consolidado (leito) sob condições darcianas/não-darcianas em uma grande faixa de número de Reynolds. Uma nova abordagem de modelagem foi proposta, a qual inclui a condutividade molecular em paralelo com a permeabilidade. Os resultados mostram uma forte correlação dos experimentos com o modelo proposto, em que o aumento da velocidade do fluido, em condições turbulentas, aumenta o efeito da resistência viscosa inercial, diminuindo a condutividade molecular. Analisando o modelo de efeito inercial de Forchheimer (1901) percebe-se, matematicamente, que este pode ser desdobrado na abordagem de condutividade paralela proposta neste trabalho.
\end{abstract}

\section{INTRODUÇÃO}

O escoamento de fluidos em meios porosos é uma situação encontrada em larga escala nos segmentos da indústria assim como no meio ambiente. Uma propriedade essencial ao escoamento é a permeabilidade, que é dependente de características do meio poroso como: porosidade, tortuosidade e tamanho e forma das partículas. A permeabilidade é a propriedade macroscópica do meio poroso que caracteriza a facilidade com que os fluidos podem escoar através do corpo sólido poroso (Bear, 1972; Dias et al., 2007).

O estudo de fenômeno de transporte em meios porosos iniciou-se com Henry Darcy, que obteve a clássica expressão que leva seu nome através de observações experimentais (Darcy, 1856)

$$
U=-\frac{k}{\mu} \Delta p_{0}
$$

onde $U, \Delta p_{0}, \mu$ e $k$ representam, respectivamente a velocidade média, o gradiente de pressão, a viscosidade do fluido e a permeabilidade. A lei de Darcy estabelece que existe uma propriedade do 
meio que independe da relação diretamente proporcional entre o gradiente de pressão e a vazão de fluido, sendo esta válida somente em condições de velocidade baixa (Scheidegger, 1960; Bear, 1972; Dullien, 1979).

Os regimes de escoamentos viscosos são classificados em laminar ou turbulento. No regime laminar o escoamento é caracterizado pelo movimento em camadas bem definidas por linhas de corrente. No regime turbulento o escoamento é caracterizado por movimentos moleculares tridimensionais aleatórios em adição ao movimento médio do fluido. A classificação de um escoamento (laminar ou turbulento), usualmente, é obtida através do cálculo do número de Reynolds (Bear, 1972).

Zeng e Grigg (2003) aplicaram uma modelagem físico-matemática para revisar os critérios de número de Reynolds e número de Forchheimer utilizados na obtenção dos limites de transição do escoamento em meios porosos. O critério de Forchheimer foi recomendado devido ao claro significado das variáveis envolvidas. Como o escoamento de fluidos em meios porosos ocorre quase sempre em regime de turbulência, pesquisas têm sido realizadas a fim de observar os desvios da lei de Darcy. Vários termos como fluxo não-darciano, fluxo inercial, efeito da resistência viscosa inercial, etc., são usados para descrever este comportamento (Firoozabadi e Katz, 1979). Em vários trabalhos foram sugeridas correções na equação de Darcy (Tek, 1957; Geertsma, 1974). Forchheimer (1901) adicionou um termo de segunda ordem com relação à velocidade para representar o efeito inercial microscópico, levando à clássica equação de Forchheimer

$$
\frac{d P}{d L}=\frac{\mu v}{k}+\beta \rho v^{2}
$$

onde $P$ é a pressão, $L$ é a direção que o fluido escoa, $\mu$ é a viscosidade do fluido, $v$ é a velocidade, $k$ é a permeabilidade, $\beta$ é o coeficiente não-darciano e $\rho$ a densidade do fluido. Uma revisão com diversos modelos propostos para explicar os desvios não lineares da equação de Darcy podem ser encontrados em Muskat (1946).

Dias et al. (2007), além de modelar, realizou experimentos e simulações computacionais para analisar a permeabilidade em um meio poroso bifásico. Fazendo o uso da equação de KozenyCarman, baseado-se em experimentos, sugeriram um modelo para mensurar os efeitos do empacotamento dos meios porosos abordados.

O objetivo do presente trabalho é apresentar uma nova abordagem de modelagem, juntamente com experimentos, no estudo do efeito viscoso inercial junto à permeabilidade de um meio poroso não consolidado sob escoamento não compressível. Neste considera-se a condutividade molecular em paralelo com a permeabilidade absoluta do meio poroso em condições darcianas e não-darcianas. Os resultados experimentais foram analisados e comparados com a abordagem de modelagem proposta. 


\section{MATERIAIS E MÉTODOS}

\subsection{Aparato Experimental}

Para o estudo do escoamento de água em um meio poroso não consolidado sob condições darcianas e não-darcianas, desenvolveu-se um aparato experimental equivalente a um permeâmetro. $\mathrm{O}$ esquema do permeâmetro é apresentado na Figura 1. O detalhe do meio poroso não consolidado (esferas de vidro de $3 \mathrm{~mm}$ ) pode ser observado na Figura 2.

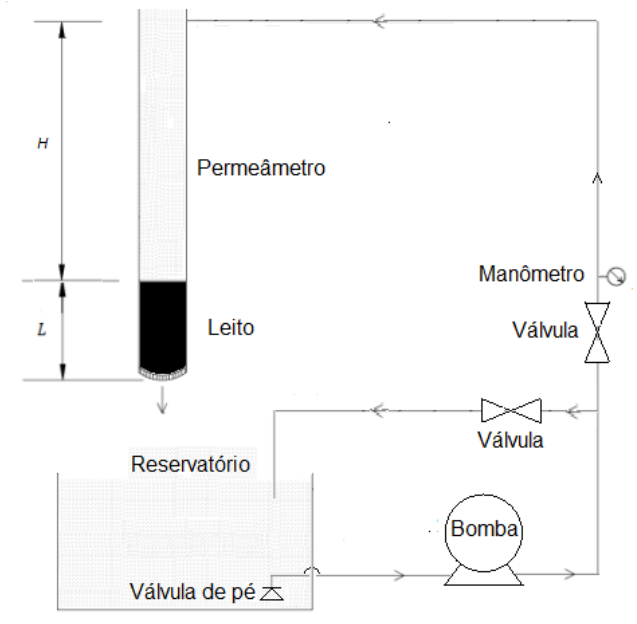

Figura 1 - O esquema do permeâmetro

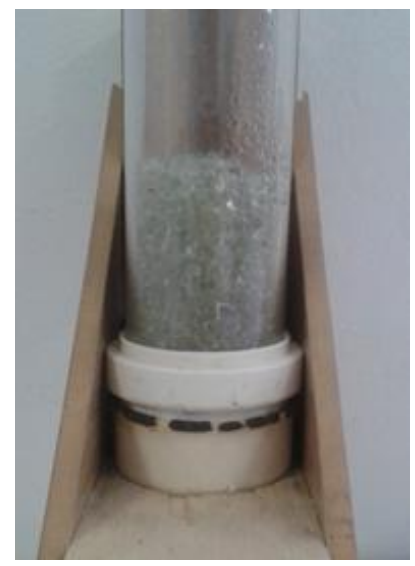

Figura 2 - Leito formado por esferas de vidro

Este esquema consiste basicamente em tubulações de seção circular e suas respectivas conexões, um tubo de acrílico de seção circular (preenchido com esferas de vidro), duas válvulas, uma peneira metálica, uma bomba, um manômetro e um reservatório. Para a realização do experimento foi necessário a utilização de um cronômetro, termômetro e trena.

No tubo de acrílico foram inseridas as esferas de vidro de $3 \mathrm{~mm}$ de diâmetro para a formação do leito. A altura deste variou de $10 \mathrm{~cm}$ a $19,5 \mathrm{~cm}$.

Num primeiro momento calculamos a porosidade $(\varnothing)$, que é uma propriedade importante do meio poroso (Dullien, 1979), através da razão entre o volume de poros $\left(V_{p}\right)$ e o volume total do leito $\left(V_{t}\right)$. Na Tabela 1 é apresentada a permeabilidade correlacionada com a porosidade em solos com distintos tamanhos de partículas. 
Tabela 1 - Propriedades do solo

\begin{tabular}{|c|c|c|c|}
\hline Tipo de solo & Permeabilidade $\left(\mathbf{m}^{\mathbf{2}}\right)$ & Porosidade $(\boldsymbol{\%})$ & $\begin{array}{c}\text { Tamanho das } \\
\text { partículas }(\mathbf{m m})\end{array}$ \\
\hline Argiloso & $10^{-18}-10^{-15}$ & $40-70$ & $<0,002$ \\
\hline Sedimentoso & $10^{-16}-10^{-12}$ & $35-50$ & $0,002-0,05$ \\
\hline Arenoso & $10^{-14}-10^{-10}$ & $25-50$ & $0,05-2$ \\
\hline Cascalho & $10^{-10}-10^{-7}$ & $25-40$ & $>2$ \\
\hline
\end{tabular}

Para iniciar o experimento, acionou-se a bomba e, em seguida, abriu-se a válvula (1). No momento em que se alcançou a altura $H$ de coluna de água desejada acionou-se a válvula (2) para estabilização desta. Posteriormente, colocou-se uma proveta na saída do permeâmetro a fim de recolher o volume de fluido escoado durante o período de tempo cronometrado. Durante o cálculo da vazão do fluido, foi verificada, por meio de um termômetro, a temperatura do mesmo. De posse desta, determinou-se a viscosidade do fluido através da interpolação simples da tabela de propriedade da água do Fox et al. (2006).

Para o cálculo da permeabilidade, foram plotados gráficos pressão x vazão para cada experimento. A pressão responsável pelo escoamento do fluido no meio poroso foi obtida através da coluna d'agua acima do leito. Foram realizados 4 experimentos, cuja a altura $H$ dos leitos eram iguais a $10 \mathrm{~cm}, 15 \mathrm{~cm}, 18 \mathrm{~cm}$ e 19,5 cm. Para cada experimento foram realizadas de 4 a 10 medições

\subsection{Abordagem de Modelagem}

A nova abordagem de modelagem presente neste trabalho é baseada na equação de Forchheimer. Foi realizado um desenvolvimento matemático da equação clássica de Forchheimer (Equação 2) de forma a torna-la equivalente à equação de Darcy, porém, no lugar da permeabilidade $k$ obtivemos uma condutividade global equivalente $k_{e q}$, que engloba a condutividade molecular e a permeabilidade absoluta $k$.

Primeiramente, acrescentou-se e substituiu-se alguns termos, gerando a Equação 3:

$$
\frac{d P}{L}=\frac{\mu Q}{K A}+\frac{\beta \rho v Q}{A}
$$

Colocando-se alguns termos em evidencia e invertendo a equação 3 nos leva a:

$$
\frac{Q}{d P}=\frac{A}{L}\left(\frac{k}{\mu+k \beta \rho v}\right) \Rightarrow \frac{Q}{d P}=\frac{A}{\mu L}\left[\frac{k\left(\frac{\mu}{\beta \rho v}\right)}{\left(\frac{\mu}{\beta \rho v}\right)+k}\right] \Rightarrow \frac{Q}{d P}=\frac{A}{\mu L}\left(\frac{k \delta}{k+\delta}\right)
$$


que corresponde, por analogia, à clássica equação de Darcy (Equação de Darcy modificada) substituindo a permeabilidade absoluta pela condutividade global equivalente $\left(k_{e q}\right)$.

$$
\frac{Q}{\Delta P}=\frac{k_{e q} A}{\mu L}
$$

em que o termo entre parênteses na Equação 4 representa a condutividade molecular $\delta$, igual a $\delta=\mu / \beta \rho v$.

Analisando os termos e a estrutura da Equação 4, observa-se que a permeabilidade $k$ encontrase em paralelo com a condutividade molecular $(\delta)$, que é função do efeito viscoso inercial. Com isso chega-se à Equação 5, que apresenta uma condutividade global equivalente $k_{e q}$ resultante dos efeitos do meio poroso e moleculares relativos ao fluido. $\mathrm{O}$ efeito em paralelo entre as condutividades pode ser visualizado na Fig. 3, percebendo-se um comportamento assintótico, na Figura 4, entre o $k_{e q}$ e a condutividade molecular $(\delta)$.

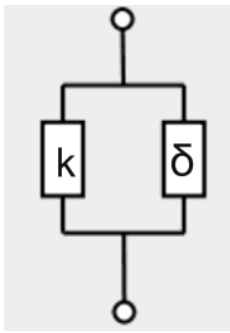

Figura 3 - Esquema condutividades em paralelo

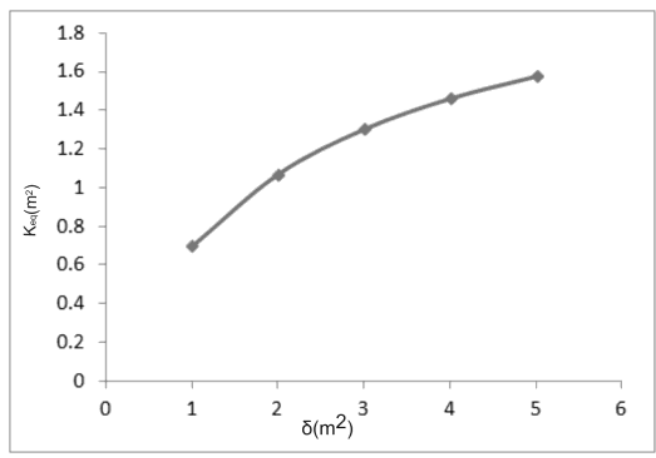

Figura 4 - Comportamento do $\mathrm{k}_{\mathrm{eq}} \mathrm{x} \delta$

A propriedade em questão $\left(k_{e q}\right)$ é resultante do efeito em paralelo da permeabilidade com a condutividade molecular, sendo somente considerada em regimes não-darcianos. Vale lembrar que em regimes darcianos o efeito viscoso inercial é desprezível, portanto a propriedade de condutividade molecular é desconsiderada, tornando o $k_{e q}$ igual à permeabilidade do leito $\left(k_{e q}=k\right)$.

\section{RESULTADOS E DISCUSSÕES}

Os resultados apresentados nesta seção compreendem o experimento realizado com o leito de comprimento $L$ igual a $18 \mathrm{~cm}$. Como descrito no tópico 2.1, o primeiro dado calculado no experimento foi a porosidade do leito sendo o valor obtido igual a $38 \%$. Analisando a Tabela $1 \mathrm{e}$ considerando o tamanho das esferas de vidro do leito, pode-se estimar que a permeabilidade do leito encontrava-se na faixa de $10^{-10}$ à $10^{-7} \mathrm{~m}^{2}$ (Veja Tabela 1$)$. 
A Tabela 2 a seguir apresenta os resultados experimentais obtidos em diversas condições de número de Reynolds.

Tabela 2 - Condições experimentais

\begin{tabular}{|c|c|c|c|c|}
\hline $\mathbf{N}^{\mathbf{o}}$ & $\begin{array}{c}\text { Pressão }(\boldsymbol{P}) \\
{[\mathbf{P a}]}\end{array}$ & $\begin{array}{c}\text { Vazão }(\boldsymbol{Q}) \\
{\left[\mathbf{m}^{3} / \mathbf{s}\right]}\end{array}$ & $\begin{array}{c}\text { Velocidade }(\boldsymbol{v}) \\
{[\mathbf{m} / \mathbf{s}]}\end{array}$ & Reynolds $\left(\boldsymbol{R}_{\boldsymbol{e}}\right)$ \\
\hline 1 & 441,5 & $5,52 \times 10^{-5}$ & 0,096 & 1686,9 \\
\hline 2 & 588,6 & $5,68 \times 10^{-5}$ & 0,099 & 1737,3 \\
\hline 3 & 833,9 & $6,24 \times 10^{-5}$ & 0,108 & 1905,9 \\
\hline 4 & 1128,2 & $6,54 \times 10^{-5}$ & 0,114 & 1999,1 \\
\hline 5 & 1275,3 & $6,76 \times 10^{-5}$ & 0,118 & 2067,7 \\
\hline 6 & 1471,5 & $6,96 \times 10^{-5}$ & 0,121 & 2126,6 \\
\hline 7 & 1667,7 & $7,04 \times 10^{-5}$ & 0,123 & 2152,1 \\
\hline 8 & 1863,9 & $7,32 \times 10^{-5}$ & 0,127 & 2237,5 \\
\hline 9 & 2452,5 & $7,67 \times 10^{-5}$ & 0,133 & 2344,1 \\
\hline 10 & 3090,2 & $8,10 \times 10^{-5}$ & 0,141 & 2475,3 \\
\hline
\end{tabular}

Analisando-se os dados da Tabela 2, nota-se que o experimento foi realizado no regime nãodarciano. De acordo com a Equação 5, as variáveis $k_{e q}, A, \mu e L$ compõem o coeficiente angular em termos do gráfico vazão vs pressão. A Figura 3 seguinte apresenta os dados experimentais, em termos de vazão-pressão, em diversos regimes de escoamento, a fim de obtermos o coeficiente angular da reta. O coeficiente angular das retas foi determinante na obtenção da condutividade global equivalente $\left(k_{e q}\right)$.

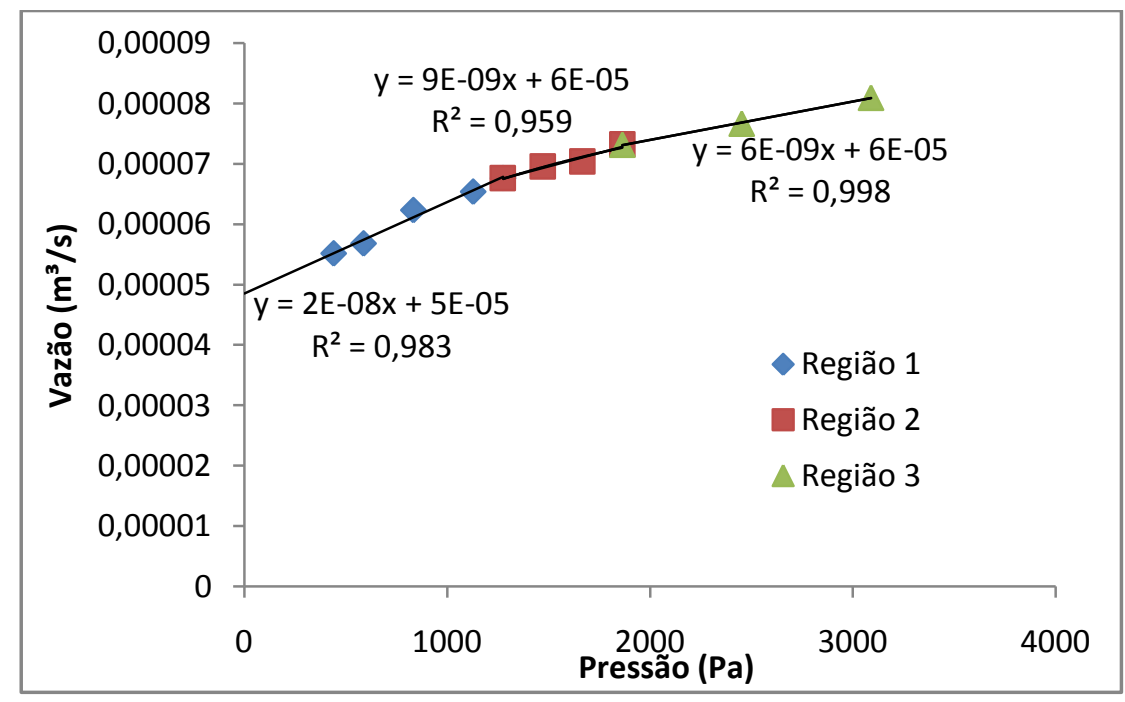

Figura 5 - Gráfico vazão x pressão do experimento 


\section{9 a 22 de outubro de 2014 \\ Florianópolis/SC}

Com a plotagem dos dados, identificamos a existência de regiões com coeficientes angulares distintos. Visando obter o melhor coeficiente de correlação para a linha de tendência linear, dividimos os dados experimentais em três regiões com sua respectiva linha de tendência linear.

A linha de tendência da região 1 , apesar de iniciar em $P=0$, apresenta um coeficiente linear não nulo, o que é fisicamente coerente, já que o gradiente de pressão aplicado pela coluna d'agua é zero. Porém, se observarmos a Figura 3, nota-se que existe um resido de água preenchendo o leito em $H=0$, o que é suficiente para gerar escoamento do fluido devido ao efeito gravitacional sobre a massa de fluido nos poros.

Como as propriedades $A, \mu e L$ foram obtidas a priori e os coeficientes angulares foram obtidos nas equações das linhas de tendências apresentadas no gráfico, calculou-se o $k_{e q}$ em cada uma destas. A Tabela 3 apresenta os valores do $k_{e q}$ de cada região. Comparando os valores da condutividade global equivalente das três regiões, notou-se a diminuição do valor de acordo com o aumento do gradiente de pressão externo aplicado. Isto ocorreu devido ao aumento do efeito viscoso inercial presente no escoamento. Na nova abordagem de modelagem proposta no presente trabalho, a condutividade molecular $(\delta)$, atua em paralelo com a permeabilidade do leito, conforme a Equação 4. Para correlacionar o experimento com a modelagem, considerou-se desprezível o efeito viscoso inercial na região 1 do gráfico da Figura 3, portanto, $k_{e q}=k$ na região 1 . A partir desta consideração, calculou-se o $\delta$ das demais regiões. Para obter o coeficiente não-darciano $(\beta)$ da equação de cada região, foi necessário calcular a velocidade média das medições pertencentes a esta. As demais propriedades foram encontradas a priori. A Tabela 3 apresenta os valores de $k, \delta, \beta$, velocidade media $\left(v_{m}\right)$ e $k_{e q}$ para suas respectivas regiões.

Tabela 3 - Propriedades do experimento

\begin{tabular}{|c|c|c|c|c|c|}
\hline Região & $\boldsymbol{k}\left[\boldsymbol{m}^{2}\right]$ & $\boldsymbol{\delta}\left[\boldsymbol{m}^{2}\right]$ & $\boldsymbol{\beta}\left[\boldsymbol{m}^{-1}\right]$ & $\boldsymbol{v}_{\boldsymbol{m}}[\boldsymbol{m} / \boldsymbol{s}]$ & $\boldsymbol{k}_{\boldsymbol{e q}}\left[\mathbf{m}^{2}\right]$ \\
\hline 1 & $2,30 \times 10^{-9}$ & 0 & 0 & $1,07 \times 10^{-1}$ & $2,30 \times 10^{-9}$ \\
\hline 2 & $2,30 \times 10^{-9}$ & $1,88 \times 10^{-9}$ & $4,26 \times 10^{3}$ & $1,22 \times 10^{-1}$ & $1,04 \times 10^{-9}$ \\
\hline 3 & $2,30 \times 10^{-9}$ & $0,986 \times 10^{-9}$ & $7,42 \times 10^{3}$ & $1,34 \times 10^{-1}$ & $6,90 \times 10^{-10}$ \\
\hline
\end{tabular}

Analisando a Tabela 3 e admitindo-se que a permeabilidade do leito atua em paralelo com a condutividade global, pode-se constatar que a diminuição da condutividade global equivalente $\left(k_{e q}\right)$ ocorreu devido a diminuição da condutividade molecular do fluido $(\delta)$, oriundo do aumento do efeito viscoso inercial. Em relação ao coeficiente não-darciano, pode-se observar que a velocidade de escoamento do fluido, provavelmente, interfere em seu valor.

\section{CONCLUSÃO}

Os resultados apresentam uma forte correlação do experimento com a nova abordagem de modelagem proposta no presente trabalho. O desenvolvimento matemático da equação de Forchheimer mostrou que o escoamento de fluidos incompressíveis em meios porosos sob condições não-darcianas apresenta a condutividade global equivalente como resultante dos efeitos do meio 
poroso em paralelo com os efeitos moleculares relativos ao fluido. Ao analisar os resultados experimentais percebeu-se que a condutividade molecular segue uma tendência direta com a condutividade global equivalente sob condições não-darcianas. Analisando o gráfico observou-se a presença de regiões de transição lineares com elevados coeficientes de correlação no ajuste dos dados experimentais junto à equação de Darcy modificada. Em relação ao coeficiente não-darciano, pode-se observar uma forte relação deste com a velocidade média do fluido.

\section{REFERÊNCIAS}

BEAR, J., Dynamics of fluids in porous media. New York: Editora American Elsevier, 1972.

DARCY, H. Les fontaines publiques de la ville de Dijon, Dalmont, Paris, 1856.

DIAS, R.P.; FERNANDES, C.S.; TEIXEIRA, J.A.; MOTA, M.; YELSHIN, A. Permeability and effective thermal conductivity of bisized porous media, Journal of Hydrology, v. 349, p. 470474, 2008.

DULLIEN, F. A. L. Porous media: fluid transport and pore structure. San Diego: Editora Academic Press, 1992.

FIROOZABADI, A.; KATZ, D. L. An analysis of high-velocity gas flow through porous media, J. Petrol. Technol, p. 211-216, 1979.

FORCHHEIMER, P. Wasserbewegung durch boden, Zeit. Ver. Deutsch. v. 45, p. 1781-1788, 1901.

FOX, R. W; MCDONALD, A. T.; PRITCHARD, P. J. Introdução à mecânica dos fluidos. Rio de Janeiro: Editora LTC, 2006.

GEERTSMA, J. Estimating the coefficient of inertial resistance in fluid flow through porous media, Soc. Petrol. Eng. J. p. 445-450, October 1974.

MUSKAT, M. The Flow of Homogeneous Fluids through Porous Media. The Mapple Press Company, 1946

SCHEIDEGGEAR, E. The Physics of Flow through Porous Media. Toronto: Editora Macmillan, 1960.

TEK, M. R. Development of a generalized Darcy equation, Trans. AIME. v. 210, p. 376-377, 1957.

ZENG, Z.; GRIGG, R. A Criterion for Non-Darcy Flow in Porous Media, Transport in Porous Media. v. 63, p. 57-69, 2006. 